\title{
Changes in left ventricular twist after mitral valve repair
}

\author{
Toshinobu Kazui, MD, ${ }^{a}$ Hiroyuki Niinuma, MD, ${ }^{\mathrm{b}}$ Junichi Tsuboi, MD, ${ }^{\mathrm{c}}$ and Hitoshi Okabayashi, $\mathrm{MD}^{\mathrm{c}}$
}

Objective: We performed echocardiographic tissue tracking to investigate whether mitral valve repair preserves left ventricular function.

\begin{abstract}
Methods: We studied 16 subjects without heart disease (11 male; mean age, $54.6 \pm 15.1$ years) and 18 patients in normal sinus rhythm undergoing solitary mitral valve repair (12 male; mean age, $53.6 \pm 16.6$ years). Transthoracic echocardiography was performed before and after surgery, and left ventricular apical and basal short-axis images were recorded. Left ventricular rotation angle was measured with off-line Vector Velocity Imaging (Siemens Medical Solutions USA Inc, Mountain View, Calif) at each slice level.

Results: Left ventricular ejection fraction was significantly higher in the control $(68.4 \% \pm 3.6 \%)$ and preoperative groups $(70.9 \% \pm 6.5 \%)$ than the postoperative group $(59.4 \% \pm 11.4 \%, P<.05)$. Left ventricular enddiastolic and end-systolic volumes were significantly greater in the preoperative group than the control group $(130.0 \pm 41.5 \mathrm{~mL}$ and $41.6 \pm 16.6 \mathrm{~mL}$ vs $80.0 \pm 16.7 \mathrm{~mL}$ and $26.6 \pm 9.2 \mathrm{~mL}$, respectively, $P<.05)$. Left ventricular end-diastolic volume normalized postoperatively. Left ventricular twist was significantly greater in the preoperative group than the other groups $\left(11.7^{\circ} \pm 4.1^{\circ}\right.$ versus $7.1^{\circ} \pm 3.8^{\circ}$ and $\left.8.2^{\circ} \pm 5.7^{\circ}, P<.05\right)$. Left ventricular twist did not differ significantly between control and postoperative groups. New York Heart Association functional class improved from $1.6 \pm 0.5$ to $1.0 \pm 0.0$ after surgery $(P<.05)$.
\end{abstract}

Conclusions: Although preoperative left ventricular ejection fraction seemed normal, left ventricular twist was greater. Left ventricular twist normalized after surgery, suggesting that it preserves left ventricular function. (J Thorac Cardiovasc Surg 2011;141:716-24)

Mitral valve repair (MVP) is considered the criterion standard for surgical repair of nonrheumatic mitral valve insufficiency. MVP has a low incidence of reoperation and complications and preserves left ventricular (LV) function. ${ }^{1}$ In addition, MVP is superior to mitral valve replacement in terms of long-term survival. ${ }^{2}$ Preservation of the mitral valve complex, which consists of the leaflet, annulus, chordae, and papillary muscle, plays an important role in preserving LV function after MVP; however, the complete reason that MVP is better at preserving LV function remains unclear. ${ }^{3}$

Measuring LV twist has been possible but difficult; however, recent studies on echocardiographic tissue tracking have brought another perspective to this LV function. ${ }^{4}$ To understand LV twist, Taber and colleagues ${ }^{5}$ proposed a model of helical layer architecture composed of obliquely aligned muscle fibers. It showed that on the one hand the contraction of the epicardial fibers will rotate the LV apex in counterclockwise and the base in clockwise direction,

From Heart Center, ${ }^{a}$ Department of Cardiovascular Surgery, St. Luke's International Hospital; and the Division of Cardiology, Department of Medicine, ${ }^{b}$ and Department of Cardiovascular Surgery, ${ }^{\mathrm{c}}$ Memorial Heart Center, Iwate Medical University, Tokyo, Japan.

Disclosures: Authors have nothing to disclose with regard to commercial support.

Received for publication June 28, 2009; revisions received April 7, 2010; accepted for publication May 9, 2010; available ahead of print June 11, 2010.

Address for reprints: Toshinobu Kazui, MD, Cardiovascular Surgery, St. Luke's International Hospital, 9-1 Akashicho Chuoku, Tokyo 104-8560, Japan (E-mail: t-kazui@pf6.so-net.ne.jp).

0022-5223/\$36.00

Copyright $(c) 2011$ by The American Association for Thoracic Surgery doi:10.1016/j.jtcvs.2010.05.004 whereas on the other hand contraction of subendocardial region will rotate the LV apex and base in exactly the opposite direction. We can now easily assess LV twist with the 2-dimensional (2D) tracking method.

Many studies have claimed that MVP is superior to mitral valve replacement in preserving cardiac function. ${ }^{6,7}$ These studies have reported that MVP reconstructs or preserves the mitral apparatus, which may be a major reason for the preservation of LV twist after surgery. Perioperative changes in LV twist have remained unclear, however, so this study aimed to examine these changes in LV twist and cardiac function before and after MVP.

\section{MATERIALS AND METHODS Study Population}

Thirty-four adults were enrolled, and the data were acquired from June 2006 to October 2008. All subjects provided informed consent before undergoing clinical examination to evaluate valvular heart disease or exclude other cardiac disease. The control group consisted of 16 subjects ( 11 men and 5 women, mean age $54.6 \pm 15.1$ years), and their inclusion criteria were as follows: (1) no evidence of structural cardiovascular disease by 2D echocardiography, (2) LV ejection fraction (LVEF) greater than $60 \%$ according to the modified Simpson method, (3) no evidence of atrial fibrillation, (4) no evidence of bundle branch block, and (5) normal blood pressure. The MVP group consisted of 18 patients ( 12 men and 6 women; mean age, $53.6 \pm 16.6$ years) who underwent MVP because of nonrheumatic, pure degenerative mitral regurgitation (MR). The mean MR grade was $3.9 \pm 0.3$ (grade 3-4). The patients in this group had no other underlying diseases, with well-compensated degenerative MR, and underwent only MVP. As shown in Table 1, there were no significant differences between the control and MVP groups in age or body surface area. This study was approved by the institutional review board of our hospital. 


$$
\begin{aligned}
& \text { Abbreviations and Acronyms } \\
& \begin{array}{ll}
\text { 2D } & =2 \text {-dimensional } \\
\text { CRT } & =\text { cardiac resynchronization therapy } \\
\text { HR } & =\text { heart rate } \\
\text { LV } & =\text { left ventricle } \\
\text { LVEDV } & =\text { left ventricular end-diastolic volume } \\
\text { LVEF } & =\text { left ventricular ejection fraction } \\
\text { LVESV } & =\text { left ventricular end-systolic volume } \\
\text { MR } & =\text { mitral regurgitation } \\
\text { MVP } & =\text { mitral valve repair } \\
\text { SV } & =\text { stroke volume } \\
\text { VVI } & =\text { Vector Velocity Imaging }
\end{array}
\end{aligned}
$$

\section{Surgical Procedures}

Our surgical indication was chronic, severe MR with a simple lesion in otherwise symptom-free patients with good LV function. Because of our extensive experience in this field, we believe that these patients were suitable for the operation.

The surgical procedures are listed in Table 2. All patients underwent median sternotomy and standard cardiopulmonary bypass under normothermic conditions. They also underwent an annuloplasty. Seven of the 18 patients underwent an artificial chordae procedure. The operations were uneventful, and no major postsurgical complications occurred. To maintain consistency, concomitant and MVP procedures that affected LV dimensions were not performed.

\section{Echocardiography}

All subjects uneventfully underwent standard echocardiography before and after surgery. Echocardiography was performed by an experienced technician. LVEF (as a percentage), LV end-diastolic dimension (in centimeters), LV end-systolic dimension (in centimeters), LV end-diastolic volume (LVEDV in milliliters), LV end-systolic volume (LVESV in milliliters), LV stroke volume (SV in milliliters), and effective regurgitant orifice area (in square millimeters) were evaluated before and after surgery. Heart rate (HR) and systolic and diastolic blood pressures were also measured for accurate evaluation of hemodynamic condition and MR. Cardiac output $(\mathrm{CO}$, in milliliters per minute) was calculated as $\mathrm{SV} \times \mathrm{HR}$. Echocardiography was performed 1 month before surgery and approximately 2 weeks after surgery. The 2D data sets of the $2 \mathrm{LV}$ short-axis planes at apical and basal levels were acquired with harmonic gray-scale imaging with a commercially available ultrasound transducer (3V2c or 4V1c, Sequoia c512; Siemens Medical Solutions USA Inc, Mountain View, Calif). We used our internal landmarks to acquire the proper short-axis view such that the basal shortaxis plane contained the mitral valve and the apical plane was acquired distally to the papillary muscles. As van Dalen and associates ${ }^{8}$ reported, if the apical short-axis image is obtained near the midventricular level, LV apical rotation may be significantly underestimated. We therefore changed the transducer position to 2 intercostal spaces more caudal, as van Dalen and associates ${ }^{8}$ previously reported. At each plane, 3 consecutive cardiac cycles were acquired at a frame rate of $42 \mathrm{~Hz}$ during breath holding, and the images were digitally stored on a hard drive. These images were exported to a personal computer and were analyzed off-line with customized software.

\section{Vector Velocity Imaging Analysis}

The off-line Vector Velocity Imaging (VVI) software (Syngo; Siemens Medical Solutions) provided regional functional information by using a tracking algorithm to estimate myocardial velocity at a set of points on a contour in a 2D sequence of B-mode images (Figure 1). Endocardial borders were manually identified in a single cineloop frame, and the borders in other frames were automatically generated, thus enabling the operator to alter any of these contours. The borders were tracked throughout the cardiac cycle by the speckle tracking method. The direction of the vector velocity indicated the direction in which the tissue was moving. Because circumflex velocity indicated the rotation of a point, VVI measured a rotation angle that was calculated from circumflex velocity with the center of the LV as a reference point. The average rotation velocities of both $L V$ basal and apical endocardium were obtained with off-line VVI analysis. Any counterclockwise rotation, as viewed from the LV apex, was expressed as a positive value, whereas any clockwise rotation was expressed as a negative value (Figure 1). After this analysis, depicted basal and apical LV rotation data points were exported to a spreadsheet program (Excel; Microsoft Corp, Seattle, Wash) to calculate LV twist. To adjust for intersubject differences in $\mathrm{HR}$, the time sequence was normalized to the percentage of systolic duration. End systole was considered to be the point of mitral valve opening according to the M-mode. LV twist was calculated as the difference between apical and basal rotation angles. Maximum LV twist values were compared among the 3 groups.

To evaluate diastolic function of the myocardium, we evaluated LV untwist (as rotation angle per second). Maximum LV untwist values were also compared among the 3 groups.

\section{Statistical Analysis}

The variables are presented as mean $\pm \mathrm{SD}$. The degrees of $\mathrm{LV}$ rotation and LV twist and untwist were compared among the three groups with analysis of variance. Differences between the control and preoperative groups and between the control and postoperative groups were tested with 2-tailed Student $t$ tests for unpaired data. The preoperative and postoperative groups were compared with paired $t$ tests. Repeated-measures analysis of variance was done to evaluate the mean changes of twist angle, twist, and untwist angle speed during the time course. These analysis were done with Stat View (version 5.0; SAS Institute Inc, Cary, NC).

\section{RESULTS}

All operations were successful, and postoperative MR grade improved to trivial MR (Table 1). The New York Heart Association functional class improved significantly from $1.6 \pm 0.5$ before surgery to $1.0 \pm 0.0$ after surgery $(P<.05)$.

Changes in preoperative and postoperative maximum twist are shown in Figure 2. All but 6 patients had decreased maximum twist after surgery. Maximum LV twist was found in the preoperative MVP group (Figure 3, $A$ ). The maximum values were $7.1^{\circ} \pm 3.8^{\circ}, 11.7^{\circ} \pm 4.1^{\circ}$, and $8.2^{\circ} \pm 5.7^{\circ}$ in the control, preoperative MVP, and postoperative MVP groups, respectively $(P<.05)$. LV twist curve in the preoperative MVP group was also higher than that of either the control or postoperative MVP group (Figure 4, A).

$\mathrm{HR}$ and systolic and diastolic blood pressures were also determined to evaluate hemodynamic performance (Table 1). Postoperative HR was significantly faster than control and preoperative HRs $(85.6 \pm 6.8$ beats/min vs $73.6 \pm 7.6$ beats/min and $85.6 \pm 6.8$ beats/min vs $66.3 \pm 8.2$ beats/ $\mathrm{min}$, respectively, $P<.05$ ). Systolic and diastolic blood pressures were higher in the control group than in the preoperative and postoperative groups, except there was no significant difference in diastolic blood pressure between the control and postoperative groups. 
TABLE 1. Patient characteristics

\begin{tabular}{|c|c|c|c|c|}
\hline & Group A & Group B & Group C & $P$ value \\
\hline No. & 16 & 18 & - & \\
\hline Sex (male/female) & 11:5 & $12: 6$ & - & \\
\hline Age $(y$, mean $\pm S D)$ & $54.6 \pm 15.1$ & $53.6 \pm 16.6$ & - & NS \\
\hline New York Heart Association functional class (mean \pm SD) & - & $1.6 \pm 0.5$ & $1.0 \pm 0.0$ & B vs $\mathrm{C}<.05$ \\
\hline Height $(\mathrm{cm}$, mean $\pm \mathrm{SD})$ & $155.0 \pm 39.3$ & $164.7 \pm 10.1$ & - & NS \\
\hline Body weight $(\mathrm{kg}$, mean $\pm \mathrm{SD})$ & $59.9 \pm 10.5$ & $58.3 \pm 11.9$ & $59.4 \pm 11.4$ & NS \\
\hline Body surface area $\left(\mathrm{m}^{2}\right.$, mean $\left.\pm \mathrm{SD}\right)$ & $1.62 \pm 0.18$ & $1.63 \pm 0.18$ & $1.64 \pm 0.18$ & NS \\
\hline Heart rate (beats/min, mean $\pm \mathrm{SD}$ ) & $73.6 \pm 7.6$ & $66.3 \pm 8.2$ & $85.6 \pm 6.8$ & $\begin{array}{l}\text { A vs } B .0112 \\
\text { A vs } C<.0001 \\
B \text { vs } C<.0001\end{array}$ \\
\hline Systolic blood pressure $(\mathrm{mm} \mathrm{Hg}$, mean $\pm \mathrm{SD})$ & $121.6 \pm 6.2$ & $114.1 \pm 8.9$ & $112.2 \pm 9.6$ & $\begin{array}{l}\text { A vs B } .0085 \\
\text { A vs C } .0022 \\
\text { B vs C NS }\end{array}$ \\
\hline Diastolic blood pressure $(\mathrm{mm} \mathrm{Hg}$, mean $\pm \mathrm{SD})$ & $69.8 \pm 6.3$ & $62.9 \pm 8.0$ & $71.6 \pm 9.1$ & $\begin{array}{l}\text { A vs B NS } \\
\text { A vs C } .0090 \\
\text { B vs C } .0004\end{array}$ \\
\hline LV ejection fraction $(\%$, mean $\pm \mathrm{SD})$ & $68.4 \pm 3.6$ & $70.9 \pm 6.5$ & $60.6 \pm 9.2$ & $\begin{array}{l}\text { A vs B NS } \\
\text { A vs C } .0028 \\
\text { B vs C } .0006\end{array}$ \\
\hline LV end-diastolic dimension $(\mathrm{cm}$, mean $\pm \mathrm{SD})$ & $4.5 \pm 0.4$ & $5.5 \pm 0.5$ & $4.5 \pm 0.4$ & $\begin{array}{l}\text { A vs } \mathrm{B}<.0001 \\
\mathrm{~B} \text { vs } \mathrm{C}<.0001 \\
\mathrm{~A} \text { vs } \mathrm{C} \text { NS }\end{array}$ \\
\hline LV end-systolic dimension $(\mathrm{cm}$, mean $\pm \mathrm{SD})$ & $2.9 \pm 0.4$ & $3.2 \pm 0.4$ & $3.0 \pm 0.4$ & $\begin{array}{l}\text { A vs B } .0197 \\
\text { A vs C NS } \\
\text { B vs C NS }\end{array}$ \\
\hline LV end-diastolic volume (mL, mean $\pm \mathrm{SD})$ & $80.0 \pm 16.7$ & $130.0 \pm 41.5$ & $95.4 \pm 42.4$ & $\begin{array}{l}\text { A vs B } .0001 \\
B \text { vs C } .0051 \\
\text { A vs C NS }\end{array}$ \\
\hline LV end-systolic volume $(\mathrm{mL}$, mean $\pm \mathrm{SD})$ & $26.6 \pm 9.2$ & $41.6 \pm 16.6$ & $38.1 \pm 16.4$ & $\begin{array}{l}\text { A vs B } .0043 \\
\text { A vs C } .0227 \\
\text { B vs C NS }\end{array}$ \\
\hline Intraventricular septal thickness $(\mathrm{cm}$, mean $\pm \mathrm{SD})$ & $0.9 \pm 0.1$ & $1.1 \pm 0.2$ & $1.2 \pm 0.2$ & $\begin{array}{l}\text { A vs B } .0106 \\
\text { A vs } C<.0001 \\
\text { B vs C NS }\end{array}$ \\
\hline Posterior wall diameter $(\mathrm{cm}$, mean $\pm \mathrm{SD})$ & $1.0 \pm 0.1$ & $1.1 \pm 0.2$ & $1.2 \pm 0.2$ & $\begin{array}{l}\text { A vs B } .0006 \\
\text { A vs } C<.0001 \\
\text { B vs C NS }\end{array}$ \\
\hline Mitral regurgitation grade (mean $\pm \mathrm{SD}$ ) & - & $3.9 \pm 0.3$ & $0.5 \pm 0.6$ & $\mathrm{~B}$ vs $\mathrm{C}<.0001$ \\
\hline Effective regurgitant orifice area $\left(\mathrm{mm}^{2}\right.$, mean $\left.\pm \mathrm{SD}\right)$ & - & $66.7 \pm 36.4$ & $1.1 \pm 1.8$ & $\mathrm{~B}$ vs $\mathrm{C}<.0001$ \\
\hline Early/late ventricular filling velocity ratio (mean $\pm \mathrm{SD}$ ) & & $1.9 \pm 1.0$ & $1.5 \pm 0.7$ & NS \\
\hline Cardiac output $(\mathrm{L} / \mathrm{min}$, mean $\pm \mathrm{SD})$ & $4.0 \pm 0.8$ & $6.0 \pm 2.1$ & $5.0 \pm 2.3$ & $\begin{array}{l}\text { A vs } B .0010 \\
\text { A vs } C<.1174 \\
\text { B vs } C<.0102\end{array}$ \\
\hline Stroke volume $(\mathrm{mL}$, mean $\pm \mathrm{SD})$ & $54.2 \pm 9.1$ & $90.9 \pm 30.0$ & $57.8 \pm 25.6$ & $\begin{array}{l}\text { A vs } \mathrm{B}<.0001 \\
\mathrm{~A} \text { vs } \mathrm{C} \text { NS } \\
\mathrm{B} \text { vs } \mathrm{C}<.001\end{array}$ \\
\hline
\end{tabular}

Group A, Control; Group B, preoperative mitral valve repair; Group $C$, postoperative mitral valve repair; $N S$, not significant; $L V$, left ventricular.

LVEF did not differ significantly between the control and preoperative MVP groups (Table 1). Postoperative LVEF values were, however, lower than preoperative LVEF values $(P<.05)$. Postoperative LVEDV, which was smaller than preoperative LVEDV, was almost identical to that of the control group $(95.4 \pm 42.4 \mathrm{~mL})$. Preoperative and postoperative LVESVs were larger than the control values. Although the differences in interventricular septal thickness and poste- rior wall diameter were approximately 0.1 or $0.2 \mathrm{~cm}$, the interventricular septal thickness $(1.2 \pm 0.2 \mathrm{~cm})$ and posterior wall diameter $(1.2 \pm 0.2 \mathrm{~cm})$ were thickest in the postoperative MVP group. Effective regurgitant orifice area decreased significantly after surgery $\left(66.7 \pm 36.4 \mathrm{~mm}^{2}\right.$ vs $1.1 \pm 1.8 \mathrm{~mm}^{2}, P<.05$ ). Preoperative $\mathrm{CO}$ was significantly greater than control CO $(6.0 \pm 2.1 \mathrm{~L} / \mathrm{min}$ vs $4.0 \pm$ $0.8 \mathrm{~L} / \mathrm{min}$ ), and postoperative $\mathrm{CO}$ was decreased from 
TABLE 2. Surgical procedures

\begin{tabular}{lc}
\hline \multicolumn{1}{c}{ Procedures } & No. \\
\hline Resection suture with flexible band & 5 \\
Sliding plasty with flexible band & 3 \\
Artificial chordae with flexible band & 3 \\
Resection suture with artificial chordae and semirigid ring & 2 \\
Artificial chordae with edge-to-edge and flexible band & 2 \\
Resection suture with artificial chordae and flexible band & 1 \\
Perforation closure with c1 plication and flexible band & 1 \\
P2 plication with edge-to-edge, artificial chordae, and flexible band & 1 \\
\hline
\end{tabular}

preoperative $\mathrm{CO}(6.0 \pm 2.1 \mathrm{~L} / \mathrm{min}$ vs $5.0 \pm 2.3 \mathrm{~L} / \mathrm{min}$, $P<.05)$. CO did not differ significantly between the control and postoperative groups $(4.0 \pm 0.8 \mathrm{~L} / \mathrm{min}$ vs $5.0 \pm 2.3 \mathrm{~L} / \mathrm{min}$ ).

Changes in SV showed a pattern similar to that of LVEF. As shown in Table 1, SV was greater in the preoperative MVP group than in both the control and postoperative MVP groups $(P<0.05)$.

To investigate the different pattern of LV twist changes, we divided the postoperative group into 2 subgroups, the increased postoperative LV twist group and the decreased postoperative LV twist group (Table 2). We compared LVEF, LVEDV, and LVESV between these 2 subgroups. Although the differences were not significant, the decreased postoperative LV twist group tended to have greater LVEDV $(144.5 \pm 49.9 \mathrm{~mL}$ vs $119.8 \pm 28.2 \mathrm{~mL})$ and $\operatorname{LVESV}(47.1 \pm 19.8 \mathrm{~mL}$ vs $38.5 \pm 10.4 \mathrm{~mL})$.

Although the difference was not statistically significant, LV untwist was faster in the preoperative MVP group $\left(-91.0 \pm 54.2^{\circ} / \mathrm{s}\right)$ than in the control group $\left(-54.9 \pm 34.2^{\circ} / \mathrm{s}\right)$ and the postoperative MVP group $\left(-76.3 \pm 40.7^{\circ} / \mathrm{s}\right.$; Figure $3, B)$. The changes in early/late ventricular filling velocity ratio were not significantly different and were within normal limits (Table 1). We considered the result of LV untwist to be compatible with conventional diastolic evaluation. LV twist and untwist velocity curves also did not differ significantly among the 3 groups (Figure 4,B).

\section{DISCUSSION}

\section{Characteristics of VVI}

The concept that the helical fibers ${ }^{9}$ create cardiac muscle twist and untwist during the cardiac cycle was recently proposed and has been assessed by some studies with echocardiography and magnetic resonance imaging. ${ }^{4,10}$ This idea has shed light on the concept of twist and untwist cardiac functions, which had not been easily observed previously. Because VVI is a relatively new method of measuring myocardial velocity, intraobserver, interobserver, and testretest variabilities still remain to be verified for this method. Some studies with VVI have reported that this method is reliable in terms of reproducibility (5\%-7\% variability). ${ }^{11}$ The feasibility and reproducibility of LV rotation parameters measured by speckle tracking echocardiography with QLAB
TABLE 3. Differences between groups with increased and decreased postoperative left ventricular twist

\begin{tabular}{|c|c|c|c|}
\hline & $\begin{array}{l}\text { Increased twist } \\
\qquad(\mathrm{n}=6)\end{array}$ & $\begin{array}{l}\text { Decreased twist } \\
\quad(n=12)\end{array}$ & $P$ value \\
\hline \multicolumn{4}{|l|}{ Twist $\left(^{\circ}\right)$} \\
\hline Preoperative & $8.3 \pm 3.7$ & $13.5 \pm 3.1$ & .0062 \\
\hline Postoperative & $12.8 \pm 5.6$ & $5.9 \pm 4.3$ & .0104 \\
\hline \multicolumn{4}{|c|}{ LV ejection fraction $(\%)$} \\
\hline Preoperative & $70.0 \pm 6.8$ & $71.3 \pm 6.7$ & NS \\
\hline Postoperative & $63.0 \pm 4.9$ & $60.1 \pm 9.8$ & NS \\
\hline \multicolumn{4}{|c|}{ LV end-diastolic volume (mL) } \\
\hline Preoperative & $119.8 \pm 28.2$ & $144.5 \pm 49.9$ & NS \\
\hline Postoperative & $82.0 \pm 18.2$ & $89.8 \pm 32.5$ & NS \\
\hline \multicolumn{4}{|c|}{ LV end-systolic volume (mL) } \\
\hline Preoperative & $38.5 \pm 10.4$ & $47.1 \pm 19.8$ & NS \\
\hline Postoperative & $34.0 \pm 9.4$ & $36.5 \pm 15.9$ & NS \\
\hline
\end{tabular}

All data are mean $\pm \mathrm{SD} . L V$, Left ventricular; $N S$, not significant.

(Philips, Best, The Netherlands) were reported on by van Dalen and coworkers. ${ }^{12}$ They stated that tracking points placed midmyocardially were superior to tracking points placed endocardially or epicardially. The method and the software used are both excellent. VVI tracks the endocardium because it is easier to track. In other words, the difference between the myocardium and cardiac cavity is clear, and the epicardium tends to be affected by the lung. The endocardium is also more sensitive than the other positions in detecting an abnormal cardiac condition.

\section{The History of LV Twist}

Torsion of the LV during ejection phase was reported by Arts and colleagues ${ }^{13}$ in 1984 . They reported that 2D echocardiography validates the presence of torsion in the normal heart. Masuda and coworkers ${ }^{11}$ reported that the assessment of VVI at mitral valve opening permits easy detection of asynchronous wall motion during acute myocardial ischemia that cannot be diagnosed by conventional systolic wall thickness measurements. According to their study, sensitivity was $100 \%$ and specificity was $89 \%$ for detecting coronary artery occlusion. Thus the VVI method can measure aspects of cardiac function that were previously not measurable with other methods.

\section{The Effectiveness of LV Twist}

Echocardiographic measurement of LV twist has enabled the evaluation of asynchronous $\mathrm{LV}^{14}$ and acute myocardial ischemia ${ }^{15}$ for further studies. The studies evaluated the relationship between cardiac resynchronization therapy (CRT) and LV twist. It concluded that peak LV torsion and LV twist at aortic valve closure had the highest sensitivity and specificity for predicting patients with responses to CRT. The latter study showed that the decrease in LV twist correlated with the extent of asynergic area and global LV function. In contrast, Zang and colleagues ${ }^{16}$ reported that LV 

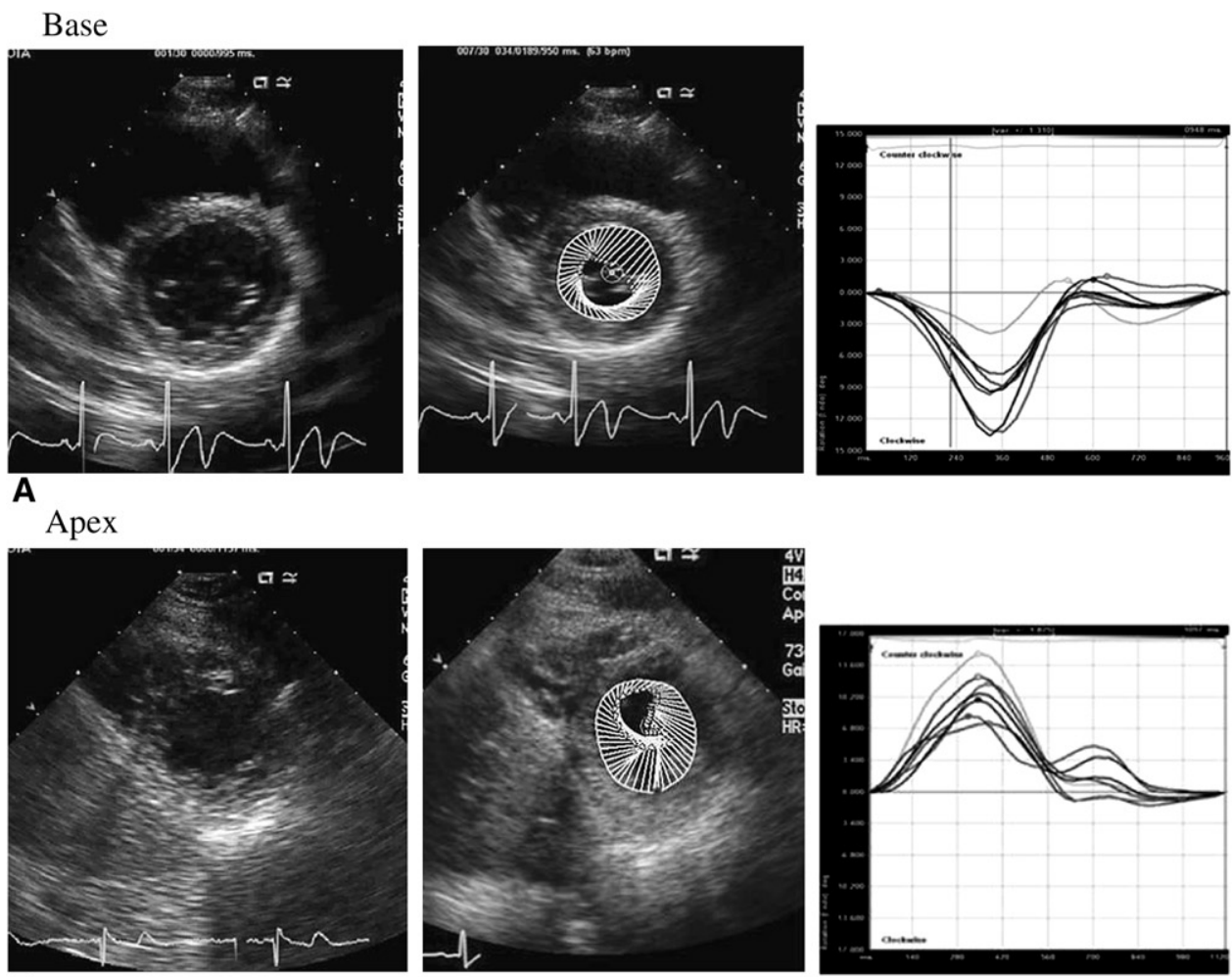

B

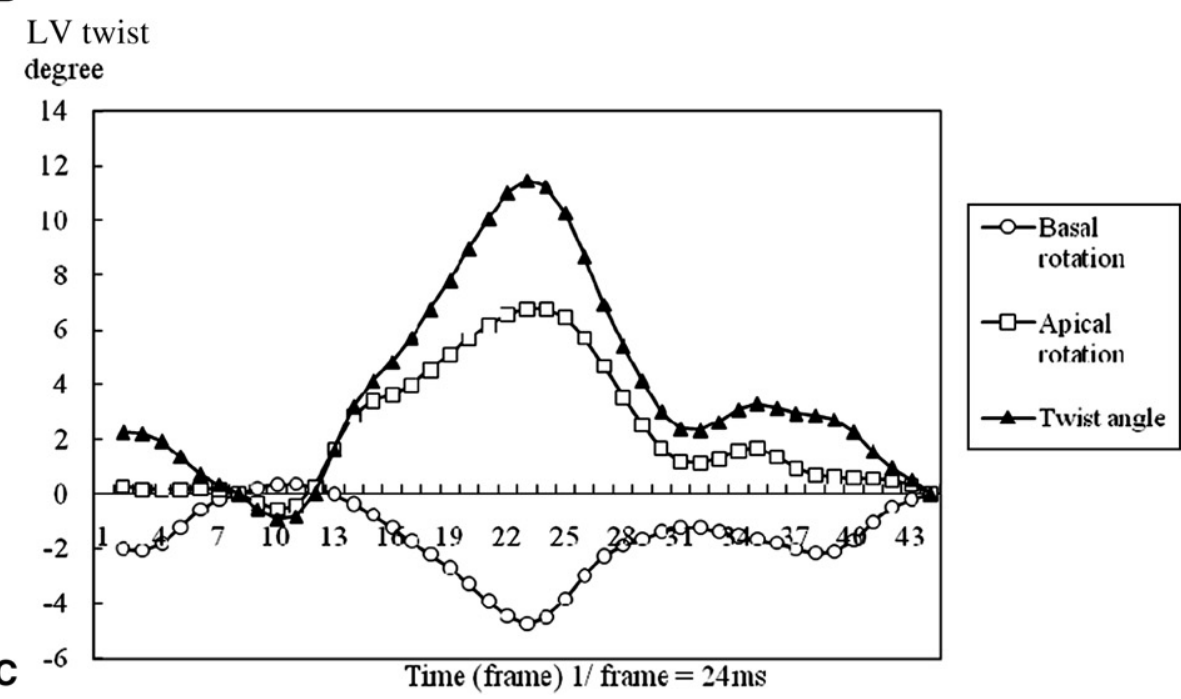

FIGURE 1. Endocardium was tracked. It was divided into 6 parts. Vector Velocity Imaging calculated angle, then average of 6 parts was calculated for base (A) and apex (B). C, Finally, left ventricular $(L V)$ twist angle was calculated by subtracting basal rotation from apical rotation.

torsion did not improve after CRT despite significant gains in LV global and short-axis function in those with responses to CRT. Those without responses showed further reduction in $\mathrm{LV}$ torsion. Considering that LV twist is mainly caused by long-axis fiber contraction that occurs in an oblique manner and in opposite directions from epicardial and endocardial muscle layers, their finding seems to be compatible with lack of improvement in LV twist after CRT. Further investigation is recommended.

\section{Originality of This Study}

Although the VVI method is unique, its main purpose in this investigation was not to assess heart valve disease. To our knowledge, this study is the first to compare LV twist before and after MVP in human patients. Some studies of LV twist, however, have used magnetic resonance imaging tagging instead of the speckle tracking method. Although these researchers investigated different diseases, some of their findings are similar to ours. Nagel and associates ${ }^{17}$ 


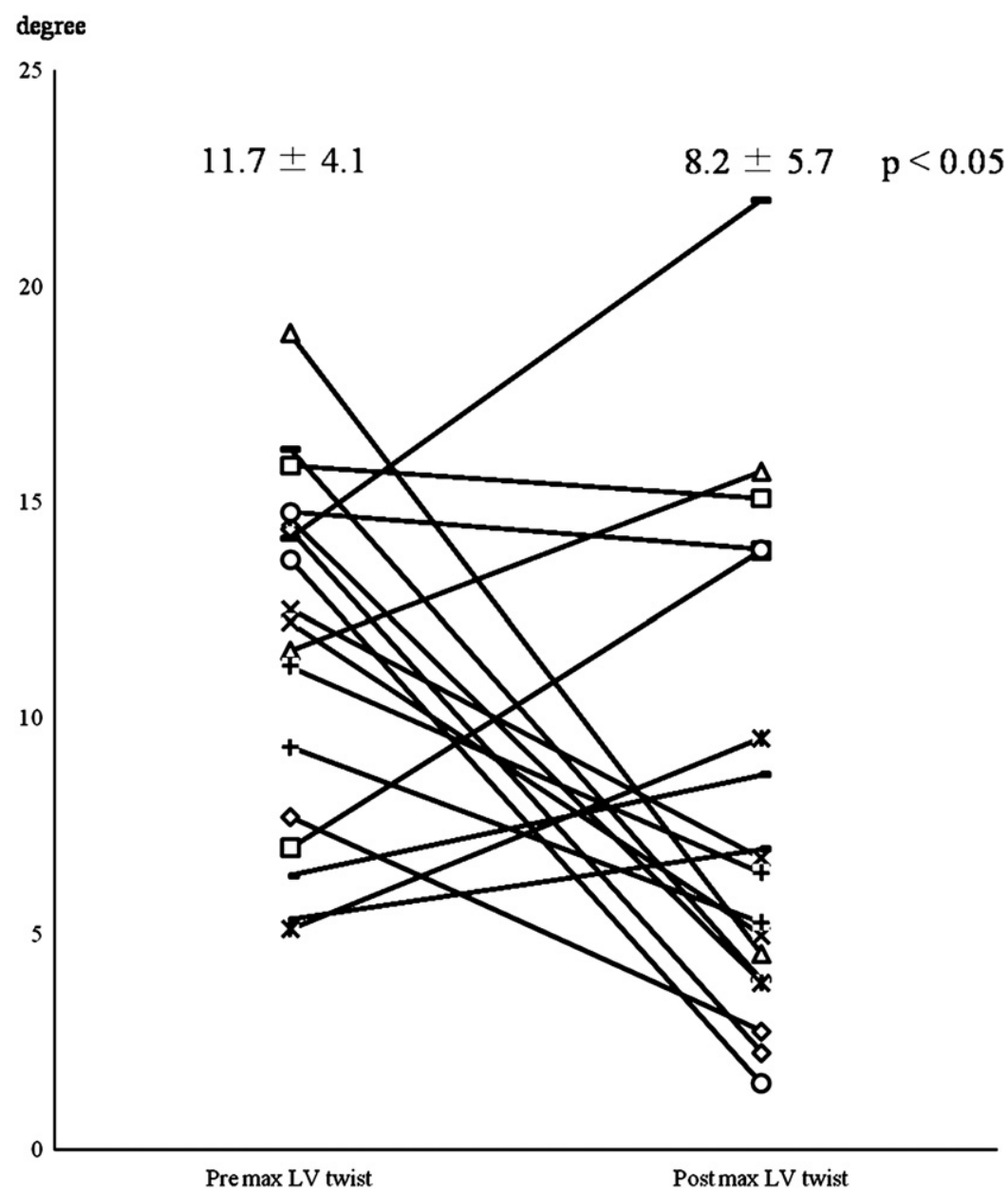

FIGURE 2. Changes in maximum left ventricular $(L V)$ twist. Pre max $L V$ twist, Preoperative maximum left ventricular twist $\left(\right.$ mean, $\left.11.7^{\circ} \pm 4.1^{\circ}\right) ; P$ ost $m a x$ $L V$ twist, postoperative maximum left ventricular twist (mean, $8.2^{\circ} \pm 5.7^{\circ} ; P<.05$ between time points).

investigated aortic valve stenosis with magnetic resonance imaging tagging. They reported that LV pressure overload was associated with increased torsion and decreased and prolonged diastolic untwisting in aortic stenosis.

\section{Function After MVP}

MVP is the standard surgical technique for treating nonrheumatic MR because the procedure is thought to preserve LV function after surgery. ${ }^{6}$ One compelling theory for the superiority of MVP is that it preserves the submitral valve apparatus, which maintains papillary-annular continuity after surgery. ${ }^{7}$ We assume that papillary-annular continuity plays an important role not only in dispersing the pressure toward the entire LV and mitral valve but also in helping LV twist and untwist to occur during the cardiac cycle.

\section{Twist in MVP}

In this study, the preoperative MVP group had the largest maximum twist value $\left(11.7^{\circ} \pm 4.1^{\circ}\right)$, indicating that $\mathrm{LV}$ twist increased along with increased SV, which covers preserved forward SV and regurgitant volume. The preoper- ative MVP group also had a larger LVEDV than did the control and postoperative MVP groups. At the same time, SV and $\mathrm{CO}$ of the preoperative MVP group were the largest among the 3 groups, and the LVEF of the preoperative MVP group did not differ significantly from that of the control group. These results suggest that the LV muscle of the preoperative MVP group had to deal with volume overload caused by MR. Increased LV twist showed this situation. Interestingly, there were 6 patients in whom LV twist increased after the operation. Although we could not find significant differences, the preoperative LVEDV tended to be larger in the decreased postoperative LV twist group $(144.5 \pm 49.9 \mathrm{~mL})$ than in the increased LV twist group $(119.8 \pm 28.2 \mathrm{~mL})$. In other words, LV twist may bear a direct relation to LVEDV in MR. We cannot draw decisive conclusion at this time, but there may be some relationship between LVEDV and LV twist. LVEF decreased after MVP. LV volume overload, LV twist, SV, and CO also decreased, nearly to the control group values. According to Suri and associates, ${ }^{18}$ surgical correction of MR results in an early decrease in LVEF, particularly in patients with 
1. Maximum LV twist

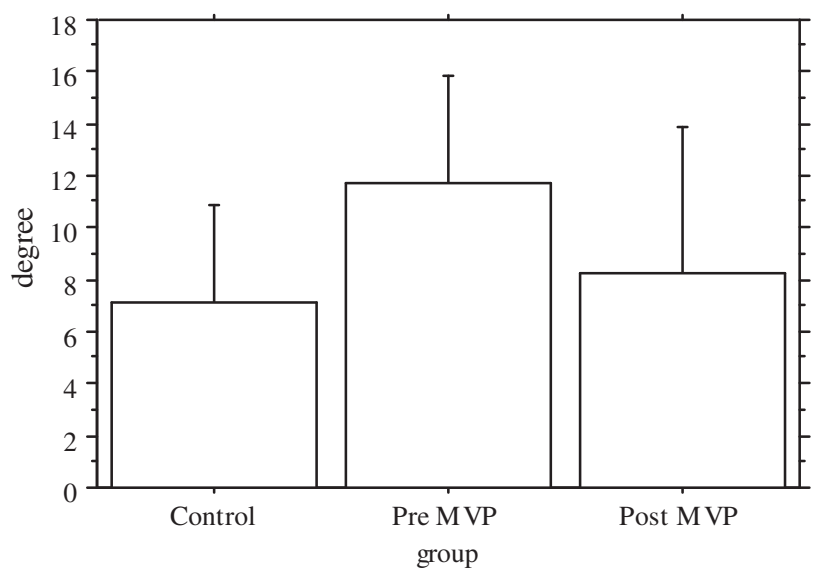

2. Maximum LV untwist

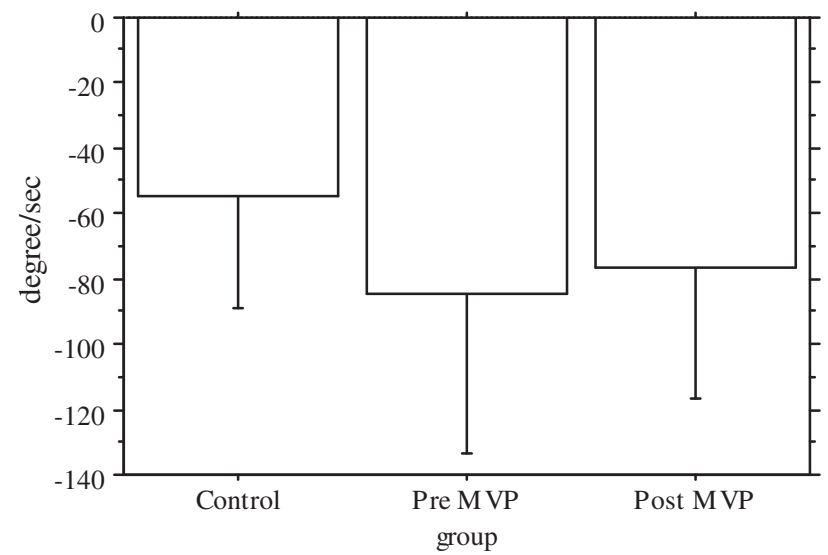

FIGURE 3. A, Maximum left ventricular $(L V)$ twist. Pre $M V P$, Preoperative mitral valve repair group; Post MVP, postoperative mitral valve repair group. Control group, $7.1^{\circ} \pm 3.8^{\circ}$. Preoperative mitral valve repair group, $11.7^{\circ} \pm 4.1^{\circ}$. Postoperative mitral valve repair group, $8.2^{\circ} \pm 5.7^{\circ}$. Control versus preoperative, $P<.05$. Control versus postoperative not significant. Preoperative versus postoperative, $P<.05$. B, Maximum left ventricular $(L V)$ untwist. Control group, $-54.9 \pm 34.2^{\circ} / \mathrm{s}$. Preoperative mitral valve repair group (Pre MVP), $-91.0 \pm 54.2^{\circ} / \mathrm{s}$. Postoperative mitral valve repair group (Post MVP), $-76.3 \pm 40.7^{\circ} / \mathrm{s}$. Control versus preoperative not significant. Control versus postoperative not significant. Preoperative versus postoperative not significant.

symptoms and increased left heart dimensions. They reported that the change in LVEF during the early postoperative period was $-8.8 \% \pm 10.7 \%$, which was similar to our results. We consider this to have occurred because preoperative regurgitant volume diminished almost completely after the operation. Our data also showed that postoperative $\mathrm{HR}$ was faster than preoperative $\mathrm{HR}$, suggesting that $\mathrm{CO}$ recovery was enough to decrease LV twist.

\section{Untwist in MVP}

LV untwist is thought to be an indicator of LV diastolic function. Some researchers have evaluated diastolic function
LV twist

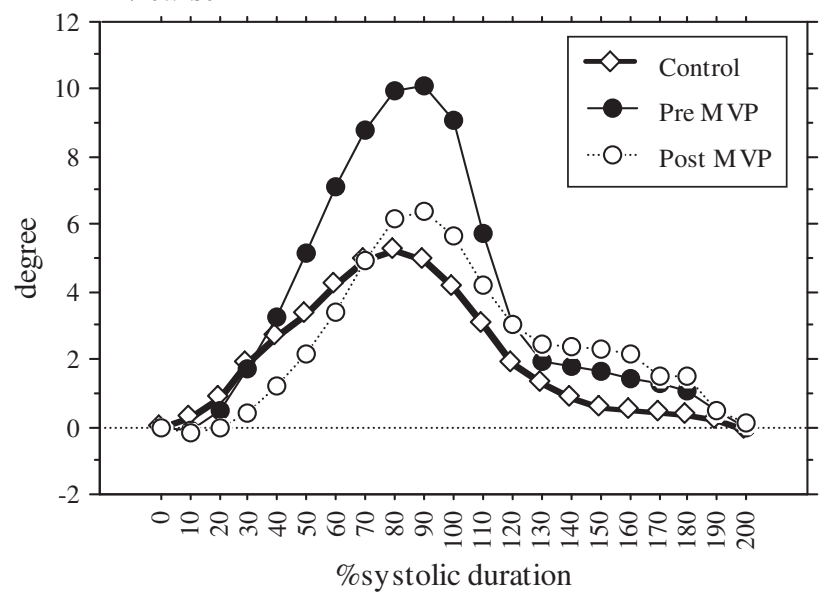

LV twist and untwist velosity

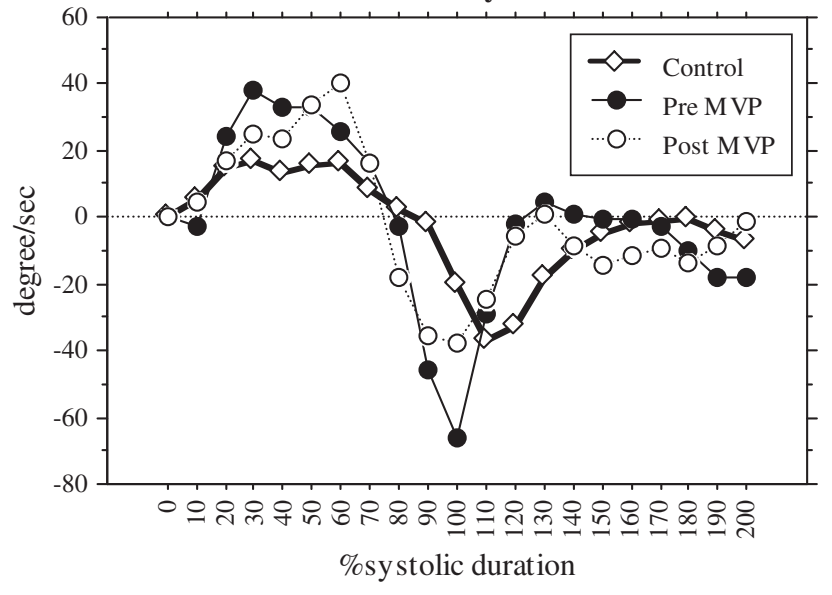

FIGURE 4. A, Left ventricular $(L V)$ twist. Preoperative mitral valve repair group (Pre MVP) versus postoperative mitral valve repair group (Post MVP) $P<.05$. Control versus preoperative not significant. B, Left ventricular twist and antitwist velocity. Preoperative mitral valve repair group (Pre MVP) versus postoperative mitral valve repair group (Post MVP) not significant. Control versus preoperative not significant.

with LV untwist by speckle tracking. ${ }^{19}$ LV untwist starts early during the isovolumic relaxation phase and proceeds throughout the early filling phase, releasing elastic energy stored by the preceding systolic deformation. LV untwist may be a useful diastolic function marker or even serve as a therapeutic target for improving diastolic function. According to Takeuchi and coworkers, ${ }^{19}$ diastolic function is impaired in hypertension and LV hypertrophy; however, we did not observe a significantly decreased untwist in this study. We assume that a reason for this finding was that the study subjects did not have LV hypertrophy and hypertension. LV twist augmentation also normally leads to augmented LV untwist. Although the effects of progression of MR and operative invasiveness on LV untwist are vague, MR and operative invasiveness did not impair LV untwist after the operation. Considering the nature of LV twist, 
preoperative and postoperative untwist were thought to be significantly different. Burns and colleagues ${ }^{20}$ reported that untwisting parameters are related to LV relaxation and suction, which are necessary for normal LV diastolic filling. Presto untwisting and smooth legato filling are necessary for the normal heart. From this point of view, it was expected that preoperative LV untwist would be faster. Although, there wasn't significant difference, there was a tendency for the preoperative MVP group to have a faster LV untwist than the postoperative MVP group. In terms of diastolic function, not only LV untwist but also early/late ventricular filling velocity ratio showed no significant difference between the preoperative and postoperative MVP groups. Although there was no significant difference, a study involving larger number of subjects is needed to verify this finding for LV untwist.

\section{The Relationship Between LVEF and LV Twist}

The relationship between LVEF and LV twist is still unclear. We found that when LVEF increased, LV twist also increased preoperatively. When LVEF decreased, LV twist also decreased postoperatively. We cannot clearly state whether the relationship between LVEF and LV twist is linear, however, nor whether there is any causal relationship. Tibayan and associates ${ }^{21}$ reported that progression from acute to chronic MR is accompanied by decreased and delayed systolic LV torsional deformation and a decrease in early diastolic recoil, which may contribute to LV dysfunction. Kanzaki and coworkers ${ }^{22}$ reported that systolic torsion amplitude was impaired in proportion to LV function in patients with dilated cardiomyopathy and poor LV function (LVEF $27 \% \pm 8.0 \%$ ). Although their study suggested that $\mathrm{LV}$ twist decreases as LV function deteriorates, we assume that during the early stages of chronic MR, when good LV function is maintained, LV twist does not decline. In another study, Notomi and associates ${ }^{23}$ showed that LV torsion and untwisting velocity increase with exercise. According to this study, $\mathrm{LV}$ twist still has a reserve in the early stages of chronic MR; however, LV twist may still decrease during the end stages of MR.

\section{Potential of LV Twist Measurement}

LVEF is believed to be an important indicator for surgery because it does not recover after surgery if it decreases below $55 \%{ }^{24}$ Furthermore, quantitative grading of MR is a powerful predictor of the clinical outcome of asymptomatic MR. ${ }^{25}$ We cannot clearly state that changes in LV twist in a failing heart are caused by chronic severe MR; however, changes in LV twist might provide additional information regarding the correct timing for surgery.

\section{Study Limitations}

Because the study included a small number of subjects, the MVP procedures were not identical. Additional studies with larger numbers of subjects are needed. In addition, although the echocardiography technician had extensive experience, the $2 \mathrm{D}$ plane may have been somewhat oblique, thereby leading to different results with the VVI software. Repetition of this clinical investigation with volume mechanics would be valuable. Further investigation is needed to determine more clearly the role of LV twist.

In terms of LV untwist, this study had a power of 0.5 to show a statistically significant difference of $50^{\circ} / \mathrm{s}$ or more $(P=.05)$. According to Takeuchi and coworkers,${ }^{19}$ diastolic negative velocity was $-59.4 \pm 23.4^{\circ} \%$ s. This study theoretically had a power of 0.88 to show a statistically significant difference of $-25 \%$ or more in the LV untwist with a $P$ value of .05. In our study, however, our data varied widely that there was only a power of 0.10 to verify that the difference between preoperative and postoperative untwist was significant. If $100^{\circ} / \mathrm{s}$ was the significant difference, our sample size had enough power (1.00). Thus the effect of MVP related to pure nonrheumatic MR untwist is still unclear.

\section{CONCLUSIONS}

The 2D speckle tracking imaging described here is a noninvasive assessment procedure that provides a new understanding of the effect of LV function before and after MVP. The results of this study suggest that although the relationship between LVEF and LV twist is unclear, LV twist is increased with increased LV output before MVP in patients with well-compensated MR, and LVEF and LV twist are decreased after MVP. LV untwist, which represents diastolic LV function, did not differ significantly between the study groups. In conclusion, MVP, which preserves mitral valve and LV continuity, preserved LV twist after surgery.

We thank Dr Osamu Takahashi for help in statistical preparation of the data. We also thank echocardiographic technician, Minoru Shobusawa, for his help with echocardiography.

\section{References}

1. Braunberger E, Deloche A, Berrebi A, Abdallah F, Celestin JA, Meimoun P, et al. Very long-term results (more than 20 years) of valve repair with Carpentier's techniques in nonrheumatic mitral valve insufficiency. Circulation. 2001;104 (12 Suppl. 1):I8-11.

2. Thourani VH, Weintraub WS, Guyton RA, Jones EL, Williams WH, Elkabbani S, et al. Outcomes and long-term survival for patients undergoing mitral valve repair versus replacement: effect of age and concomitant coronary artery bypass grafting. Circulation. 2003;108:298-304

3. Westaby S. Preservation of left ventricular function in mitral valve surgery. Heart. 1996;75:326-9.

4. Notomi Y, Lysyansky P, Setser RM, Shiota T, Popovic ZB, Martin-Miklovic MG et al. Measurement of ventricular torsion by two-dimensional ultrasound speckle tracking imaging. J Am Coll Cardiol. 2005;45:2034-41.

5. Taber LA, Yang M, Podszus WW. Mechanics of ventricular torsion. J Biomech. 1996;29:745-52.

6. Enriquez-Sarano M, Schaff HV, Orszulak TA, Tajik AJ, Bailey KR, Frye RL. Valve repair improves the outcome of surgery for mitral regurgitation. A multivariate analysis. Circulation. 1995;91:1022-8.

7. Ren JF, Aksut S, Lighty GW Jr, Vigilante GJ, Sink JD, Segal BL, et al. Mitral valve repair is superior to valve replacement for the early preservation of cardiac function: relation of ventricular geometry to function. Am Heart J. 1996;131:974-81. 
8. van Dalen BM, Vletter WB, Soliman OI, ten Cate FJ, Geleijnse ML. Importance of transducer position in the assessment of apical rotation by speckle tracking echocardiography. J Am Soc Echocardiogr. 2008;21:895-8.

9. Sallin EA. Fiber orientation and ejection fraction in the human left ventricle. Biophys J. 1969;9:954-64.

10. Zerhouni EA, Parish DM, Rogers WJ, Yang A, Shapiro EP. Human heart: tagging with MR imaging - a method for noninvasive assessment of myocardial motion. Radiology. 1988;169:59-63.

11. Masuda K, Asanuma T, Taniguchi A, Uranishi A, Ishikura F, Beppu S. Assessment of dyssynchronous wall motion during acute myocardial ischemia using velocity vector imaging. JACC Cardiovasc Imaging. 2008;1:210-20.

12. van Dalen BM, Soliman OI, Vletter WB, Kauer F, van der Zwaan HB, et al. Feasibility and reproducibility of left ventricular rotation parameters measured by speckle tracking echocardiography. Eur J Echocardiogr. 2009;10:669-76.

13. Arts T, Meerbaum S, Reneman RS, Corday E. Torsion of the left ventricle during the ejection phase in the intact dog. Cardiovasc Res. 1984;18:183-93.

14. Sade LE, Demir O, Atar I, Muderrisoglu H, Ozin B. Effect of mechanical dyssynchrony and cardiac resynchronization therapy on left ventricular rotational mechanics. Am J Cardiol. 2008;101:1163-9.

15. Garot J, Pascal O, Diebold B, Derumeaux G, Gerber BL, Dubois-Rande JL, et al. Alterations of systolic left ventricular twist after acute myocardial infarction. Am J Physiol Heart Circ Physiol. 2002;282:H357-62.

16. Zhang Q, Fung JW, Yip GW, Chan JY, Lee AP, Lam YY, et al. Improvement of left ventricular myocardial short-axis, but not long-axis function or torsion after cardiac resynchronisation therapy: an assessment by two-dimensional speckle tracking. Heart. 2008;94:1464-71.

17. Nagel E, Stuber M, Burkhard B, Fischer SE, Scheidegger MB, Boesiger P, et al. Cardiac rotation and relaxation in patients with aortic valve stenosis. Eur Heart J. 2000;21:582-9.
18. Suri RM, Schaff HV, Dearani JA, Sundt TM 3rd, Daly RC, Mullany CJ, et al. Determinants of early decline in ejection fraction after surgical correction of mitral regurgitation. J Thorac Cardiovasc Surg. 2008;136:442-7.

19. Takeuchi M, Borden WB, Nakai H, Nishikage T, Kokumai M, Nagakura $\mathrm{T}$, et al. Reduced and delayed untwisting of the left ventricle in patients with hypertension and left ventricular hypertrophy: a study using two-dimensional speckle tracking imaging. Eur Heart J. 2007;28: 2756-62.

20. Burns AT, La Gerche A, Prior DL, Macisaac AI. Left ventricular untwisting is an important determinant of early diastolic function. JACC Cardiovasc Imaging. 2009;2:709-16

21. Tibayan FA, Yun KL, Fann JI, Lai DT, Timek TA, Daughters GT, et al. Torsion dynamics in the evolution from acute to chronic mitral regurgitation. J Heart Valve Dis. 2002;11:39-46.

22. Kanzaki H, Nakatani S, Yamada N, Urayama S, Miyatake K, Kitakaze M. Impaired systolic torsion in dilated cardiomyopathy: reversal of apical rotation at mid-systole characterized with magnetic resonance tagging method. Basic Res Cardiol. 2006; 101:465-70.

23. Notomi Y, Martin-Miklovic MG, Oryszak SJ, Shiota T, Deserranno D, Popovic ZB, Garcia MJ, Greenberg NL, Thomas JD. Enhanced ventricular untwisting during exercise: a mechanistic manifestation of elastic recoil described by Doppler tissue imaging. Circulation. 2006;113:2524-33.

24. Matsumura T, Ohtaki E, Tanaka K, Misu K, Tobaru T, Asano R, et al. Echocardiographic prediction of left ventricular dysfunction after mitral valve repair for mitral regurgitation as an indicator to decide the optimal timing of repair. J Am Coll Cardiol. 2003;42:458-63.

25. Enriquez-Sarano M, Avierinos JF, Messika-Zeitoun D, Detaint D, Capps M, Nkomo V, et al. Quantitative determinants of the outcome of asymptomatic mitral regurgitation. $N$ Engl J Med. 2005;352:875-83. 